NUTRITION

New Protein Foods

from a Correspondent

THE conference at the University of Reading, from July 13 to 15 , on the topic of new protein foods differed from many others on the same subject in that it was confined to agricultural conditions in the United Kingdom. In his introduction, Professor F. Aylward (University of Reading) classified the different kinds of new protein foods and stated that a crop for the production of protein concentrates depends in practice on a combination of factors including (1) yield of protein, (2) technical problems of harvesting, extraction and processing, (3) nutritional value of protein and freedom from anti-nutrient or toxicological factors, (4) acceptability of product for direct use as a food or as a food component, and (5) economic and commercial considerations. The yield of protein depends on the percentage of protein in the crop, the total yield of crop, the actual yield of protein after harvesting, and the yield after processing.

Dr R. R. Robinson (University of Reading) described the use of bacteria, yeasts, fungi and chlorophyll-containing plants such as unicellular algae. There are many difficulties to be overcome regarding the extraction of protein from these organisms and oilseeds, legumes and cereals are likely to be the chief source of protein during the next two or three decades. Dr B. J. F. Hudson (University of Reading) ventured to predict the possible increase in consumption of these foods in the United Kingdom up to AD 2000. On the basis of careful calculations by forty different experts it seems that textured proteins and similar plant protein concentrates might replace as much as a quarter of the meat by $\mathrm{AD} 2000$, or even earlier. Professor H. M. Sinclair (University of Oxford) dealt with the nutritional potential of these new protein foods. $\mathrm{He}$ emphasized the importance of ensuring a sufficient content of vitamin $B_{12}$, with fortification if necessary, and pointed out the valuable clinical work which is being carried out on vegans living only on plant foods.

Dr M. A. Crawford (Nuffield Institute of Comparative Medicine, London) emphasized the importance of other factors besides protein in the new foods and drew attention to the role of struc. tural phospholipids in the brain and other parts of the central nervous system. Both Professor Sinclair and Dr Crawford said that the correlation between too high a level of total phospholipids and nervous diseases seems to be connected with the phospholipids breaking down, but they did not state the cause.

Meat production and demands in the
United Kingdom were described by $\mathrm{Mr}$ H. F. Marks (Meat and Livestock Commission) and Dr A. M. Ashby (Unilever Ltd). Although Britain's entry into the Common Market would be expected to cause rises in meat prices probably greater than the corresponding income rises, they thought that the total consumption of meat would not fall much, although they agreed that much more consumption of these new protein foods will gradually develop. Dr W. F. Raymond (Grassland Research Institute, Hurley) suggested that the high cost of meat production might be curbed to some extent by using alternative feeding stuffs including perhaps those made from microorganisms.

Dr M. A. B. Boddington (Wye College, London) applied land use projections for the next 30 years to forecast possible developments in yields from land which might improve the present low conversion efficiency of only 6 per cent for beef and 12 per cent for pigs.

\section{REVERSE TRANSCRIPTASE \\ Templates for Caution}

from our Cell Biology Correspondent

As Speigelman in particular has of late taken great pains to emphasize, enzymatic activities detected by their ability to use synthetic RNAs and RNA/ DNA hybrids as templates for the synthesis of DNA cannot be immediately equated with the reverse transcriptases found in animal tumour viruses. The enzyme or enzymes in such viruses-and there is still some dispute as to whether or not a single enzyme molecule can complete the synthesis of a double stranded DNA from the single stranded RNA viral genome (Robinson and Robinson, Virology, 44, 457 ; 1971)-use endogenous viral RNAs, synthetic RNAs and hybrids as templates for the synthesis of DNA. But it does not inevitably follow that all enzymes which in vitro can be programmed by synthetic RNAs or hybrids to polymerize deoxynucleoside triphosphates function in vivo as authentic reverse transcriptases and have an endogenous and natural single stranded RNA template. Synthetic nucleic acids are useful tem. plates for the detailed investigation of reverse transcriptases once the enzymes and their natural templates have been identified, but the identification of a reverse transcriptase in a new situation depends on the identification of its natural template.

Indeed if the ability of an enzyme to use synthetic RNA as a template for DNA synthesis in vitro is taken to be diagnostic of its function in vivo as a reverse transcriptase, the Kornberg DNA polymerase I of Escherichia coli must be classified as a reverse transcriptase. For as long ago as 1963-4 Cavalieri and his colleagues proved that DNA polymerase I can utilize double stranded synthetic RNAs as

\title{
Oncogenicity of Cleaved Adenovirus DNA
}

Although the adenoviruses apparently cause nothing more devastating than respiratory infections in their natural hosts, at least some of them induce tumours when they are injected into newborn rodents and will transform some of the cells which they infect in vitro. Attempts to analyse genetically the oncogenes in adenovirus genomes, by the isolation of temperature sensitive mutants, seem most promising, but meanwhile Mayne, Burnett and Butler have set about the physical isolation of fragments of the simian adenovirus 7 (SA7) genome which contain these genes. Next Wednesday's Nature New Biology contains their progress report.

Having first shown that intact DNA isolated from SA7 is not only infectious but capable of inducing tumours when injected into newborn hamsters, they have now fragmented this DNA, either by sonication or by shearing it through a syringe, fractionated the fragments and tested their oncogenic potential. Molecules roughly half the size of the complete genome, which can be separated by virtue of their density into heavy and light halves, induce tumours like intact molecules. By contrast smaller fragments obtained by sonication are not oncogenic.

The induction of tumours by both heavy and light "half" molecules, but not by smaller pieces, is of course disappointing to anybody bent on physically isolating the SA7 oncogenes free of any other genetic material. For although these experiments prove that the whole genome is not essential for oncogenicity the fact that both halves of the genome have oncogenic potential may well mean that several regions of the genetic material, rather than a unique segment, contain oncogenes. Alternatively and more optimistically, it might be argued that the oncogenes are centrally located in a small segment of the genome, which, depending on the precise point of cleavage, might be included in either the heavy or the light "halves". The hybridization studies between "half" molecules and DNA extracted from the cells of tumours induced by heavy and light "halves" that Mayne et al. promise may perhaps resolve this issue. 